\title{
Impacto da heterogeneidade de variância residual na avaliação genética de rebanho Nelore no Bioma Amazônia
}

\author{
Impact of heterogeneity of residual variance on genetic estimate of Nellore cattle from \\ Amazônia bioma
}

\section{FERREIRA, Jorge Luís ${ }^{1}$; LOPES, Fernando Brito ${ }^{2}$; EVERLING, Dionéia Magda ${ }^{3}$; MINHARRO, Sílvia ${ }^{1}$; SILVA, Marcelo Correa da ${ }^{4}$; LIRA, Thaymisson Santos de'; PEREIRA, Leonardo de Sousa ${ }^{1}$}

\author{
${ }^{1}$ Universidade Federal do Tocantins, Araguaína, Tocantins, Brasil. \\ ${ }^{2}$ Embrapa Cerrados, Planaltina, Distrito Federal, Brasil. \\ 3Instituto Federal de Farroupilha, Santa Maria, Rio Grande do Sul, Brasil. \\ ${ }^{4}$ Universidade Federal de Goiás, Programa de Pós-Graduação em Ciência Animal, Goiânia, Goiás, Brasil. \\ *Endereço para correspondência: jorgeuft@gmail.com
}

\section{RESUMO}

Objetivou-se avaliar os efeitos da heterogeneidade de variância residual sobre a avaliação genética de bovinos da raça Nelore criados a pasto, provenientes do Bioma Amazônia, Brasil. Foram utilizados pesos ajustados aos 205 dias de idade, os quais foram classificados em três classes de desvio-padrão fenotípico: baixo, médio e alto. Houve aumento da variância genética aditiva direta e residual à medida que aumentaram as classes de desviopadrão fenotípico. As herdabilidades para as classes de desvio-padrão fenotípico baixo, médio e alto foram 0,$27 ; 0,33$ e 0,26 , respectivamente. As estimativas de correlações genéticas entre as classes de desvio-padrão fenotípico de baixo-médio, baixo-alta, e médioalto foram 0,$82 ; 0,35$ e 0,72 , respectivamente. As estimativas de correlação dos valores genéticos dos touros denotaram indicio de possível efeito da interação genótipo-ambiente. Houve uma grande variabilidade para os valores genéticos dos touros na classe de alto desviopadrão fenótipo.

Palavras-chave: carne bovina, cluster, correlação, herdabilidade, valor genético

\section{SUMMARY}

This study was carried out to evaluate the effects of heterogeneity of residual variance on genetic evaluation of cattle raised on pasture from Amazônia Bioma, Brazil. Adjusted weights at weaning weight (205 days of age) were sorted in three phenotypic classes: low (less than average weight), medium (between the average and the average plus one standard deviation) and high (greater than average plus one standard deviation). There was increased of genetic additive direct and residual variances to the extent that increased phenotypic classes. The heritabilities for low, medium and high phenotypic classes were 0.27 , 0.33 and 0.26 , respectively. These estimates suggest that mass selection could result in genetic gain on breeding program that use weaning weight as a selection criteria. Genetic correlations between the low and medium, low and high, and medium and high phenotypic classes were 0.82 , 0.35 and 0.72 , respectively. The estimate of correlation of the breeding values of sire shown effect of the heterogeneity of phenotypic variance on the prediction of animals breeding values. There was a major variability for the breeding values of sires at high phenotype class. Thus, we suggested the identification and selection of sires with the highest breeding values for weaning weight, according with environment in which these animals are being raised.

Keywords: breeding value, beef cattle, cluster, correlation, heritability 


\section{INTRODUÇÃO}

Os sistemas de exploração bovina no Brasil são bastante heterogêneos estando principalmente relacionados à diversidade climática, ambiental, edáfica e aspectos socioculturais. Dessa forma, os rebanhos bovinos de corte apresentam grande variabilidade de produção entre e dentro das diferentes regiões do Brasil.

Na produção animal, é importante avaliar os efeitos das variações fenotípicas e da heterogeneidade de variância residual no desempenho e avaliação genética de bovinos. Segundo Araújo et al. (2011), a avaliação genética pode ser influenciada pela precisão com que os parâmetros genéticos e ambientais utilizados são estimados, que pode ser afetada por vários fatores, sendo um deles a heterogeneidade de variância residual.

De acordo com Carneiro et al. (2006), se as variâncias diferirem entre rebanhos com condições diferenciadas de sistemas de produção, mas são assumidas como homogêneas, nas avaliações genéticas, os animais podem ser classificados erroneamente. Da mesma forma, Garrick \& Van Vleck (1987) preconizaram que quando a heterogeneidade de variâncias genética e/ou residual é ignorada pode-se ocasionar redução na precisão dos valores genéticos, com consequentemente redução na resposta à seleção.

Vários trabalhos (CARVALHEIRO et al. 2002; CARNEIRO et al. 2006; CARNEIRO et al. 2008; HERRERA et al. 2008, SARMENTO et al. 2010, ARAÚJO et al. 2011; BONAFÉ et al. 2011) preconizaram que a presença de heterogeneidade de variância entre rebanhos, nas avaliações genéticas, leva a predição viesada dos valores genéticos dos animais, bem como redução do progresso genético.

Dessa forma, objetivou-se verificar o efeito da heterogeneidade residual sobre as estimativas de (co)variâncias e parâmetros genéticos para a característica peso padronizado aos 205 dias de bovinos de corte da raça Nelore, criados a pasto no bioma Amazônia.

\section{MATERIAL E MÉTODOS}

Foram utilizados registros de pesos padronizados aos 205 dias de idade de 40.160 bovinos da raça Nelore criados a pasto no bioma Amazônia, compreendidos entre os anos de 1997 a 2007, considerando vacas com idade mínima de 14 meses e máxima de 15 anos. O conjunto de dados foi disponibilizado pela Associação Brasileira dos Criadores de Zebu (ABCZ). Três classes fenotípicas de peso aos 205 dias de idade, baseado no desviopadrão fenotípico foram criadas: i) animais com peso abaixo da média, ii) animais com peso similar à média até um desvio-padrão (abaixo ou acima), e iii) animais com peso superior a média mais dois desvio-padrão (Tabela 1). Os modelos univariado e bivariado foram utilizados, e continham os efeitos aleatórios genéticos diretos, efeitos genético maternal e os efeitos de ambiente permanente maternal, bem como os efeitos fixos de grupo de contemporâneos (fazenda, sexo, estação e ano de nascimento) e idade da vaca ao parto como covariável (efeitos linear e quadrático).

Para a estimação das (co)variâncias e parâmetros genéticos para P205, o modelo animal utilizado foi: $y=X \beta+Z_{1} a+Z_{2} m+Z_{3} p+e$, em que $\mathrm{y}$ é vetor das observações (P205); $\beta$ é o vetor dos efeitos fixos do modelo associado à matriz de incidência $\mathrm{X}$; a é $o$ vetor dos efeitos dos efeitos genéticos direto associados à matriz $Z_{1} ; m$ é o vetor de efeitos genéticos maternais 
associados a matriz de incidência $Z_{2}$; $\mathrm{p}$ é o vetor de efeitos aleatórios de ambiente permanente maternal associados à matriz de incidência $Z_{3}$; e e é o vetor de efeito aleatório residual.

Para obtenção das estimativas de (co)variâncias, empregou-se a metodologia da Máxima Verossimilhança Restrita Livre de Derivada (DFREML), por meio de modelos animal unicaráter, usando o aplicativo Multiple Trait Derivative Free Restricted Maximum Likelihood (MTDFREML), desenvolvido por Boldman et al. (1995).

Tabela 1. Número de animais (NA), de grupo de contemporâneos (GC), de touros, de animais na matriz de parentesco (A-1) e Coeficiente de variação (CV) para diferentes classes de desvio-padrão de peso padronizado aos 205 dias de idade de rebanhos Nelore do bioma Amazônia

\begin{tabular}{lccc}
\hline \multirow{2}{*}{ Item } & \multicolumn{3}{c}{ Classes de Desvio-Padrão Fenotípicos } \\
\cline { 2 - 4 } & Baixo & Médio & Alto \\
\hline NA & 13.666 & 11.409 & 15.085 \\
CG & 590 & 568 & 646 \\
Touro & 549 & 399 & 452 \\
A-1 & & 64.388 & \\
CV & 6,83 & 5,75 & 8,23 \\
\hline
\end{tabular}

Para avaliar a influência de variâncias heterogêneas nos dados sobre a classificação genética dos animais, foram realizadas análises estatísticas, para o peso padronizado aos 205 dias de idade utilizando-se o banco de dados completo. Utilizou-se o valor da mediana da distribuição dos desvios-padrão fenotípico dos rebanhos para P205 como critério para a estratificação dos rebanhos em três classes de desvio-padrão fenotípico (alto, médio e baixo).

Os touros foram ordenados com base nos seus valores genéticos em cada classe fenotípica, e a similaridade entre os ordenamentos foi averiguada utilizandose correlação de Spearman, através do procedimento CORR do pacote estatístico SAS. Para a construção dos clusters dos valores genéticos dos touros dentro de classe de desvio-padrão fenotípico, os valores genéticos foram padronizados, assumindo média zero (0) e variância um (1), pelo PROC STANDARD, (STATISTICAL ANALYSIS SYSTEM,
2002), e as comparações dos pesos médios dentro de cada unidade federativa componente do Bioma Amazônia, utilizando-se o teste Tukey $(\mathrm{p}<0.05)$. As correlações genéticas foram estimadas utilizando-se o aplicativo MTDFREML, em modelo bicaracterística, considerando todas as classes de desvio-padrão fenotípico como características diferentes.

\section{RESULTADOS E DISCUSSÃO}

Não foi observado diferenças significativas $(p<0,001)$ entre os pesos médios para os diferentes estados que integram a região norte do Brasil (Bioma Amazônia), entre as classes fenotípicas estudadas, obtendo-se como média geral valores de $147,70 \pm 11,77$; $173,29 \pm 13,06$ e $206,73 \pm 15,92$ (Tabela 2). As estimativas médias dos pesos na classe de alto desvio-padrão excederam em $28,5 \%$ e $14,9 \%$ os valores 
observados nas classes de baixo e médio desvio-padrão, respectivamente. Esses resultados são superiores aos reportados por Araújo et al. (2011).

Porém, houve diferença $(\mathrm{p}>0,001)$ dentro das classes de DP dentro de cada estado. Esses resultados sugerem inicialmente que a intensidade de seleção dentro de cada classe de desvio-padrão, parece ser bem diferenciada. De acordo com Carneiro et al. (2006), a magnitude dos efeitos da heterogeneidade de variância depende da intensidade de seleção, com reflexo direto na classificação do mérito genético dos animais.

Tabela 2. Pesos médios (P205) para as três classes fenotípicas nos diferentes estados integrantes da região Norte do Brasil

\begin{tabular}{|c|c|c|c|c|}
\hline \multirow{2}{*}{ Estados } & \multicolumn{3}{|c|}{ Classes de Desvio-Padrão Fenotípicas } & \multirow{2}{*}{ Média } \\
\hline & Baixo & Médio & Alto & \\
\hline Acre & $146.13^{\mathrm{Dc}}$ & $171.38^{\mathrm{Db}}$ & $208.67^{\mathrm{Ba}}$ & 175.39 \\
\hline Amazônia & $146.60^{\mathrm{Dc}}$ & $171.98^{\mathrm{Db}}$ & $204.12^{\mathrm{Da}}$ & 174.24 \\
\hline Rondônia & $147.74^{\mathrm{Cc}}$ & $174.34^{\mathrm{Bb}}$ & $204.17^{\mathrm{Da}}$ & 175.42 \\
\hline Pará & $149.46^{\mathrm{Ac}}$ & $173.39^{\mathrm{Cb}}$ & $209.97^{\mathrm{Aa}}$ & 177.61 \\
\hline Tocantins & $148.57^{\mathrm{Bc}}$ & $175.38^{\mathrm{Ab}}$ & $206.55^{\mathrm{Ca}}$ & 176.83 \\
\hline Média & 147.70 & 173.29 & 206.70 & - \\
\hline
\end{tabular}

Estimativas de variâncias genética direta e maternal, e variância residual apresentaram valores crescentes à proporção que as classes de desviopadrão aumentaram (Tabela 3). Verificou-se maior variação nas estimativas de variância residual, quando se comparou as classes de desvio-padrão alto com as de médio e baixo $(57,62 \mathrm{e}$ $51,78 \%$, observando-se ainda, proporcionalidade de variação entre as classes nas estimativas de variâncias genética direta e maternal, conforme pode ser observado na Tabela 4, indicando que a variabilidade genética na população é alta, sendo possível obter progresso genético por meio da seleção, no entanto, a contribuição ambiental é mais acentuada nas classes de alto desvio-padrão fenotípico, uma vez que maiores estimativas de variância residual estão associadas como menores herdabilidades.

A heterogeneidade de variância dentro de cada classe de desvio-padrão pode indicar a existência de interação genótipo ambiente (IGA), corroborando com o proposto por Araújo et al. (2008) e Araújo et al. (2011). Entretanto, esses resultados são diferentes ao reportado por Henderson (1984), que ao estudarem diferentes cenários de heterogeneidade de variâncias, admitiram que as variâncias genéticas aditivas e residuais mudassem proporcionalmente aos ambientes, porém, as herdabilidades não variassem. Campelo et al. (2003) também observaram mudanças nas estimativas de variâncias genética direta e residual, bem como da herdabilidade, em relação às diferentes classes fenotípicas, no peso aos 120 dias de idade de bovinos da raça Tabapuã. 
Assim, é necessário definir melhor e distinguir as fontes de variação ambiental, incluindo possíveis efeitos de heterocedasticidade de variâncias fenotípicas para que as estimativas de parâmetros genéticos sejam mais precisas e confiáveis. Segundo Araújo et al. (2008) e Araújo et al. (2011), a heterogeneidade de variâncias genética e/ou residual pode ocasionar a redução da precisão dos valores genéticos preditos e, consequentemente, redução da resposta à seleção. No entanto, a extensão do problema depende da causa, pois quando a heterogeneidade é causada por fatores ambientais, este exerce maior efeito na avaliação genética, que sua constituição genética. No entanto, se a heterogeneidade de variâncias é devido a fatores genéticos, então o problema pode não existir.

Tabela 3. Estimativas de variâncias genéticas direta e maternal, variância residual e herdabilidade para peso aos 205 dias (P205) nas diferentes classes fenotípica de desvio padrão

\begin{tabular}{lrcc}
\hline \multirow{2}{*}{ Parâmetros } & \multicolumn{3}{c}{ Classes Fenotípicas } \\
\cline { 2 - 4 } & Baixo & Médio & Alto \\
\hline Herdabilidade & 0,27 & 0,33 & 0,26 \\
Variância genética Direta & 39,02 & 59,51 & 88,17 \\
Variância genética Maternal & 8,42 & 12,59 & 21,67 \\
Variância Residual & 95,09 & 108,2 & 224,4 \\
\hline
\end{tabular}

A correlação genética entre as classes de baixo e médio, baixo e alto e médio e alto classes de desvios-padrão fenotípico variaram de médias a altas (Tabela 4). Observou-se que quando a classes de baixo e médio desvio-padrão são correlacionados com a classe de alto desvio-padrão a proporção de genes relacionados para as duas classes é baixa, indicando indícios da presença de interação genótipo-ambiente. Correlações genéticas menores que 0,8 indicam uma forte e importante interação genótipoambiente (ROBERTSON, 1959), em que a classificação dos animais pode variar entre os ambientes. Assim, quando a interação é considerada importante, alguns dos genes que controlam a característica em um ambiente são diferentes daqueles que a controlam em outro ambiente (NEPOMUCENO et al., 2013).
A correlação de Spearman entre a classificação dos touros nas diferentes classes de desvio-padrão fenotípico (Tabela 4) mostrou que houve mudanças nos valores genéticos dos touros para as classes de desvio-padrão, confirmando assim que a heterogeneidade de variância residual teve impacto sobre as predições dos valores genéticos dos touros.

Tanto as estimativas de correlações genéticas, como a correlação entre os valores genéticos dos touros nas diferentes classes de desvio-padrão fenotípico indicaram presença de interação genótipo-ambiente no conjunto dos dados. Assim, faz-se necessário a inclusão nos modelos de análises presença de heterocedasticidade de variância residual bem como de interação genótipo-ambiente. 
Tabela 4. Estimativas de correlação genética (acima da diagonal) entre as classes de desvio-padrão fenotípico e correlação de Spearman (abaixo da diagonal) entre os valores genéticos dos touros avaliados em cada classe de desviopadrão fenotípico

\begin{tabular}{lccc}
\hline Item & Baixo & Médio & Alto \\
\hline Baixo & & 0.83 & 0.58 \\
Médio & 0.82 & & 0.74 \\
Alto & 0.35 & 0.72 & \\
\hline
\end{tabular}

Não houve diferenças significativas $(\mathrm{p}>0,05)$ para os valores genéticos (BV) de pai para a classe de baixo, médio e alto fenótipo. As previsões BV, para as classes de baixo, médio a alto desviopadrão fenotípico foram $1,61 \pm 0,079$, $0,112 \pm 0,172$ e $1,93 \pm 2,41$, respectivamente. No entanto, foi observada variabilidade crescente com o aumento dos pesos médios, bem como maior variabilidade dos valores de predição para a classe com alto desviopadrão fenotípico (Figura 1).

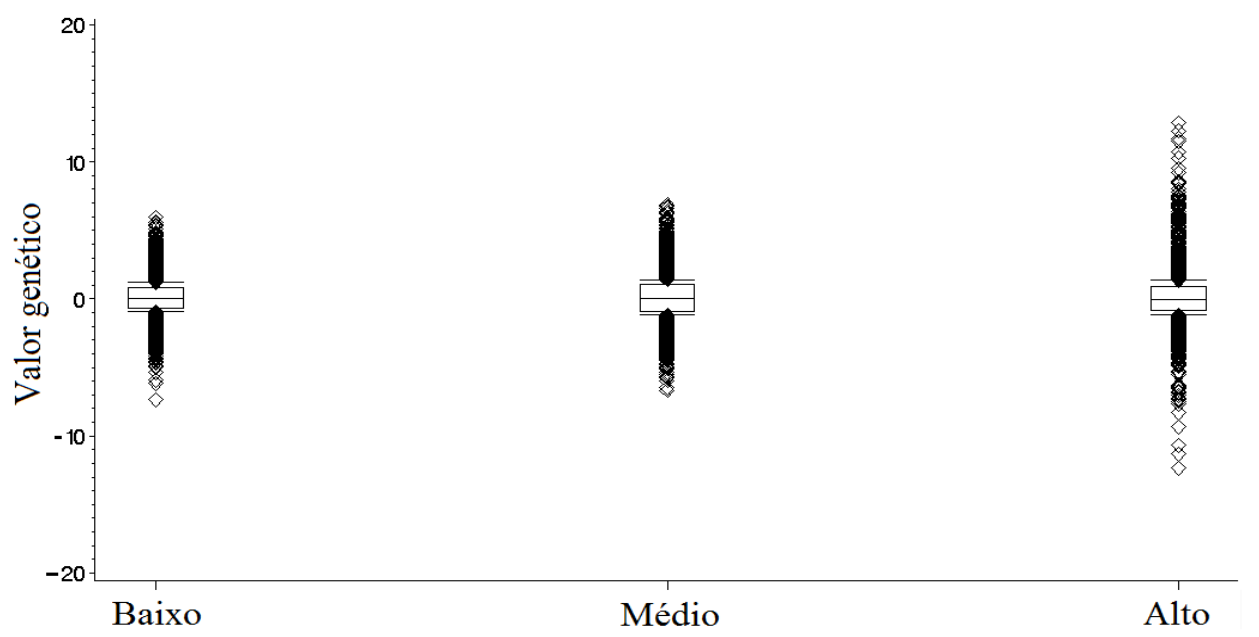

Figura 1. Valores genéticos para os touros nas diferentes classes de desvio-padrão fenotípico

Os resultados deste estudo sugerem que alguns cuidados devem ser aplicados no planejamento de um programa de melhoramento abrangente para peso ao desmame no Norte do Brasil. Uma atenção especial deve ser dada às condições ambientais em que os touros no programa foram testados, porque um pai que é satisfatório em uma região pode ter descendência com menor desempenho quando usado em outra região. Assim, o conhecimento dos efeitos de fatores ambientais/climáticos sobre os caracteres avaliados é necessário para o ajuste correto dos dados, diminuindo o viés nas informações e assim, obter resultados mais precisos, incrementando a acurácia do processo seletivo.

Assim, o reconhecimento das diferenças nos aspectos sociais, econômicos, culturais e edafoclimáticos entre as 
regiões podem aumentar a distinção entre os objetivos de seleção. A diferenciação destes objetivos são importantes para a manutenção da variabilidade genética dos animais domésticos. Dessa forma, segundo Olesen et al. (2011) não é apenas a heterogeneidade das circunstâncias da produção entre regiões, países ou explorações individuais, mas também a incerteza e $\mathrm{o}$ risco associados a circunstâncias futuras, que promoverão a diferenciação entre os objetivos de criação e manutenção de reprodutores (machos e fêmeas) mais adaptados à regiões específicas.

A implementação de programas de melhoramento genético, específico para regiões semelhantes, especialmente em relação a variáveis climáticas, é essencial para se obtiver níveis mais elevados de produção, consistentes e apropriados ao meio ambiente local. Os resultados deste estudo revelaram a existência de heterogeneidade das variâncias residuais, e quanto maior for a semelhança entre os ambientes, a variância genética direta e residual não se altera, bem como a heterocedasticidade das variâncias residuais é nula. Assim, se os efeitos da heterogeneidade das variâncias fenotípicas e ambientais não são considerados, pode haver escolha inadequada dos touros, dificultando $\mathrm{o}$ progresso genético da população sob seleção.

A heterogeneidade de variância residual interfere em parâmetros como variância genética, média fenotípica, variância fenotípica e herdabilidade, prejudicando a avaliação genética dos animais e a escolha dos pais da próxima geração. Foram verificados indicio de interação genótipo-ambiente entre as classes de baixo e alto, e médio e alto desvio padrão fenotípico. Assim, podem-se obter erros na classificação genética dos touros e consequentemente um atraso no progresso genético do rebanho.

\section{REFERÊNCIAS}

ARAÚJO, C.V.; CARDOSO, A.M.C.; RAMOS, A.A.; ARAÚJO, S.I.; MARQUES, J.R.F.; TOMAZINI, A.P.; CHAVES, L. C. Heterogeneidade de variâncias e parâmetros genéticos para produção de leite em bubalinos da raça Murrah mediante inferência Bayesiana.

Revista Brasileira de Saúde e Produção Animal [online], v.9, n.3, p.416-425, 2008.

ARAÚJO, C.V.; BTTENCOURT, T.C.B.S.C.; ARAÚJO, S.I.; LÔBO, R.B.; BEZERRA, L.A.F. Estudo da heterogeneidade de variâncias na avaliação genética de bovinos de corte da raça Nelore. Revista Brasileira de

Zootecnia, v.40, n.9, p.1902-1908, 2011.

BOLDMAN, K.G.; KRIESE, L.A.; Van VLECK, L.D. A manual for use for MTDFREML. A set of programs to obtain estimates of variance and covariance [DRAFT]. Lincoln: Department of Agriculture/Agricultural Research Service, 1995, 120p.

BONAFÉ, C.M.; TORRES, R.A. de; TEIXEIRA, R.B.; SILVA, F.G. da; SOUSA, M.F.; LEITE, C.D.S.; SILVA, L.P. da; CAETANO, G. da C.

Heterogeneidade de variância residual em Modelos de Regressão Aleatória na descrição do crescimento de codornas de corte. Revista Brasileira de Zootecnia, v.40, n.10, p.2129-2134, 2011.

CAMPELO, J.E.G.; LOPES P.S.; TORRES R.A., SILVA L.O.C.; EUCLYDES R.F.; ARAÚJO C.V.; PEREIRA C.S. Influência da heterogeneidade de variâncias na avaliação genética de bovinos de corte da raça Tabapuã. Arquivos Brasileiros de Medicina Veterinária e Zootecnia, v.55, p.685-693, 2003. 
CARNEIRO, A.P.S.; TORRES, R.A.;

LOPES, P.S.; EUCLYDES, R.F.;

CARNEIRO, P.L.S.; CUNHA, E.E.

Erros na classificação de touros, vacas e touros jovens geneticamente superiores avaliados na presença de heterogeneidade. Revista Brasileira de Zootecnia, v.35, n,5, p,1907-1914, 2006.

CARNEIRO, A.P.S.; TORRES, R.A.; LOPES, P.S.; EUCLYDES, R.F.; CARNEIRO, P.L.S.; SILVA, F.F. Heterogeneidade e avaliação genética em bovinos, estudo utilizando dados simulados. Acta Scientiarum. Animal Sciences, v.30, p.113-119, 2008.

CARVALHEIRO, R.; FRIES, L.A.; SCHENKEL, F.S.; ALBUQUERQUE, L.G. Efeitos da heterogeneidade de variância residual entre grupos de contemporâneos na avaliação genética de bovinos de corte. Revista Brasileira de Zootecnia, v.31, n.4, p.1680-1688, 2002.

GARRICK D.J.; Van VLECK, L.D. Aspects of selection for performance in several environments with heterogeneous variances. Journal of Animal Science, v.65, p.409-421, 1987.

HENDERSON, C.R. Application of linear models in animal breeding. Ontario: University of Guelf, 1984. $462 \mathrm{p}$.

HERRERA, L.G.G.; EL FARO, L.; ALBUQUERQUE, L.G. de; TONHATI, H.; MACHADO, C.H.C. Estimativas de parâmetros genéticos para a produção de leite e persistência da lactação em vacas Gir, aplicando modelos de regressão aleatória. Revista Brasileira de Zootecnia, v.37, p.15841594, 2008.
NEPOMUCENO, L.L.; LIRA, T.S.; LOPES, F.B.; LÔBO, R.B.;

FERREIRA, J.L. Interação genótipoambiente para características sob efeito maternal na raça Nelore nos estados do Maranhão, Mato Grosso e Pará. Revista Brasileira de Saúde e Produção Animal [online], v.14, n.2, p.269-276, 2013.

OLESEN, I.; GOREN, A.F.; GJERDE, B. Definition of animal breeding goals for sustainable production systems.

Journal of Animal Science, v.78, p.570-582, 2011.

ROBERTSON, A. The sampling variance of the genetic correlation coefficient. Biometrics, v.15, p.469485, 1959.

SARMENTO, J.L.R; TORRES, R.A. de; LÔBO, R.N.B.; ALBUQUERQUE, L.G. de; SOUSA, W.H. de; SOUSA, J.E.R. de. Modelos de regressão aleatória na avaliação genética do crescimento de ovinos da raça Santa Inês. Revista Brasileira de Zootecnia, v.39, n.8, p.1723-1732, 2010.

STATISTICAL ANALYSIS SYSTEM. User's guide. Version 9.0 Cary, USA:

SAS Institute, 2002.

Data de recebimento: 24/09/2013

Data de aprovação: 28/04/2014 